\title{
Towards Smart Surroundings: Enabling Techniques and Technologies for Localization
}

\author{
Kavitha Muthukrishnan \\ Maria Lijding \\ Paul Havinga \\ Fac. of Computer Science, University of Twente \\ P.O.Box 217, 7500AE Enschede, The Netherlands \\ E-mail: \{k.muthukrishnan, m.e.m.lijding, p.j.m.havinga\}@ewi.utwente.nl
}

\begin{abstract}
In this paper we identify the common techniques and technologies that are enabling location identification in a ubiquitous computing environment. We also address the important parameters for evaluating such systems. Through this survey, we explore the current trends in commercial products and research in the area of localization. Although localization is an old concept, further research is needed to make it really usable for ubiquitous computing. Therefore, we indicate future research directions and address localization in the framework of our Smart Surroundings project.
\end{abstract}

\section{Introduction}

Recent advances in wireless communication devices, sensors, hardware (MEMS) technology make it possible to envision large scale dense ad-hoc networks acting as high resolution eyes and ears of the surrounding physical space, making the vision of Mark Weiser [38] into a reality. This calm technology promises various applications and spans a wide range of fields ranging from medical and fitness, security and safety, work, learning and leisure. Ad hoc networks play a vital role in modeling these future pervasive networks. Their most distinctive features are lack of infrastructure, uncontrolled topology, and mobility. Research in ad hoc networks has become popular in the last decade with the introduction of cheap portable communication like 802.11 and Bluetooth. One aspect that was under focus from the beginning was the mobility of the network. Most basic operations in the network are directly affected by mobility: discovery of a communication party, routing, and even the very connectivity of the network.

As we approach the level of ubiquitous network con- nectivity and pervasive mobile devices, the enticing new category of context-aware applications has been proposed. By definition [7] 'context' is any information that can be used to characterize the situation of an entity. An entity is a person, place or object that is considered relevant to the interaction between a user and an application, including the user and applications themselves. A system is 'context-aware' if it uses contexts to provide relevant information and services to the user, where relevancy depends on user's tasks. One of the important dimensions of context is location. The proliferation of wireless technology, mobile computing devices and Internet has fostered a growing interest in location-aware systems and services. It is useful for accomplishing emergency services, E911, follow me services, finding the nearest resources such as printer etc, habitat monitoring, patient tracking, asset monitoring, buddy finder or a product finder, etc. Localization acts as a bridge between the virtual and physical world [8]. A mobile or a static node could answer the question 'Where am I?' in several ways. It might be relative to a map or relative to another node, or global coordinate system. This is referred to as localization or location identification.

Networked applications are often implemented in a layered architecture or protocol stack, and several layers of the protocol stack can benefit from localization [5]. Knowing the objects location not only promote context awareness at the application level, but also bumps up low level functionalities such as routing, service discovery and resource management. For instance, it augments the design of service discovery protocol, by providing seamless availability of services even while on the move.

Localization is by nature an interdisciplinary problem involving research in several areas of computer science and many kind of engineering. Consequently, research has proceeded in both the systems and the algorithmic fronts. In this paper we discuss the basic 
principles of localization, and the developments and advances made in the field. Finally, we show why localization is a corner stone in the future of pervasive computing.

\section{Performance parametric measures of localization}

Before introducing, the technologies behind the location systems, the most important thing is to have a checklist citing, the parameters that have to be kept in mind when designing such a system or algorithm. The key metric for evaluating a localization technique is the accuracy defined as, how much is the estimated position deviated from the true position. Its not just enough if the accuracy is obtained just once, it should also be consistent from measurement to measurement. For achieving high accuracy ultrasound and UWB technologies (see 1) for indoor location sensing would be of good choice. The accuracy is denoted by an accuracy value and precision value (e.g. $15 \mathrm{~cm}$ accuracy over $95 \%$ of the time). The precision indicates how often we expect to get at least the given accuracy. The accuracy of a location sensing systems is often used to determine whether the chosen system is applicable for certain applications.

Calibration also plays a very important role. The uncalibrated ranging readings are always greater than the true distance and are highly erroneous due to transmit and receive delays [39]. Device calibration is the process of forcing a device to conform to a given input/output mapping. This is often done by adjusting the device internally but can equivalently be done by passing the devices output through a calibration function that maps the actual device response to the desired response.

Responsiveness is defined as how quickly the location system outputs the location information. It is an important parameter, especially when dealing with mobility. However, this parameter is mostly ignored in the description of the existing systems.

Scalability is also significant, as the proposed design should be scalable for large-scale networks. Also of great importance is the self-organization as it is infeasible to manually configure the location determination processes for a large number of mobile devices in random configurations with random environmental characteristics.

The next most important parameter is probably the cost, which includes the cost of installation, deployment, infrastructure and maintenance. An important cost factor when running the system in a real environment is power consumption. When scaling to thou- sands or millions of autonomous small devices it is clearly not feasible to change or recharge batteries very often, thus energy efficiency should be a goal of any localization mechanism meant for a large scale system. For example in the area of wireless sensor network the goal is to provide energy autonomy to the nodes beyond three years of operation.

In our view privacy is an important parameter of localization systems and it should form part of the architecture since its conception. Using localization it is very easy to create a Big Brother infrastructure that track users movements and allow to deduce patterns of behavior. However, this issue is being generally overlooked in the design of systems and considered as an after thought only. Centralized systems are particularly weak with regard to privacy.

\section{Taxonomy of existing Location sys- tems}

Though localization is not a new field of research, it has gained additional credit due to the advent of ubiquitous computing research and its still an evolving research area, so researchers have taxonomized the localization technology in several ways. Primarily the classification can be done with respect to the environment dependability, i.e. indoor or outdoor localization systems. Examples includes in this are GPS (outdoor) [21] and [14] [29] [31] for the indoor case.

The existing location systems can also be categorized based on the type of distance measurement technique employed as: range based and range free location systems. The former systems make use of range-based measurements for measuring absolute distance/angle (e.g. Cricket [31], RADAR [29]) and the latter makes use of some other techniques that can give relative location of the objects. Range free systems [12] are of two types: using local techniques that rely on a high density of seeds so that every node can hear several seeds (e.g. Centroid algorithm and APIT), and using hop counting techniques that rely on flooding a network (e.g. DV-Hop and amorphous localization).

Researchers have also classified location systems as hardware based and software based, depending on the system implementation. Hardware-based systems need additional hardware (e.g. SpotOn [14]), while software-based systems rely on software implementations thereby reducing the cost (e.g. RADAR [29]).

Location systems can also be classified as tightly coupled or loosely coupled based on the system architecture. In the former the beacons are wired to a centralized controller and placed at fixed positions [10]. In the latter, the beacons are wireless and coordinated 
in a complete decentralized manner (e.g. Cricket, AHLoS).

Localization techniques can also be classified as centralized or distributed. The centralized approach depends on sensor nodes transmitting the data to a central server, where the computation is performed to determine the location of each node. The convex optimization techniques [20] developed by Doherty etal. and MDS-MAP [36] [35] developed by Yi Shang etal. fall into this category. These two techniques are often referred to as connectivity-based systems, because they find out the location by solving connectivity imposed proximity constraints. The distributed approach on the other hand depends on each node determining its own location using only limited communication with nearby nodes or beacons (e.g. APIT, centroid method, DV-Hop [15]).

Another way to look at the positioning algorithms is based on the type of coordinate it outputs as relative or absolute coordinate systems. An absolute coordinate system has global coherence and is desirable for most situations, being aligned to popular coordinate systems used as commercial and military references, such as GPS. These are also the most expensive in terms of communication cost and are usually based on landmarks that have known positions. Relative positioning establishes positions that are relative to a system that is local to the network and can possibly be arbitrarily, but still provide network wide coherence [24].

Yet another type of classification is the coarsegrained systems and fine-grained systems. Bulusu etal. [21] define fine grained systems as systems that obtain information by measuring the distance to a reference point using for example signal strength or timing measurement, while coarse grained systems use proximity based information for deriving the distance measurement.

\section{Enabling techniques and technolo- gies for Localization in Wireless Ad- hoc Networks}

Localization is defined as a mechanism to find the spatial relationship between objects [5]. An assumption in most of the localization system is the availability of anchor nodes or landmarks [32], while some other uses beacon nodes. Langendoen et al [19] differentiates anchor nodes and beacon nodes. They define anchor nodes as nodes having a priori knowledge of their own position with respect to some global coordinate system, while beacons (access points) are nodes based on external infrastructure (e.g. GPS-less, Cricket). Beacons have the same capabilities (processing, communication, energy consumption) as other nodes in the network. Fundamentally speaking, location systems needs some kind of input, for example it can be sensor reading originating from a sensor, or information from an access point as signal strength, for getting a symbolic representation. This information is then combined using a given technique to derive the location, either absolute or relative, of an object or set of objects. An absolute location system may use a shared reference grid for all located objects. For example, all GPS receivers use latitude, longitude and altitude for reporting the location, where as a relative location system has its own frame of reference. The following subsections give an overview about the existing technologies and techniques that enable localization.

\subsection{Signal technologies}

The different types of signal technologies are tabulated in Table 1. Depending on the required range, propagation speed, cost, precision, bandwidth etc one can choose the required technology for a specific application. As you can see from Table 1, there are infrared based, ultrasonic based, electromagnetic based, optical based, inertial based and radio frequency based systems. Depending on the type of frequency range used, Radio frequency can be categorized into RFID (Radio frequency Identification), WLAN (IEEE 802.11b), Bluetooth (IEEE 802.15), wide area cellular and UWB.

RFID is a means of storing and retrieving data through electromagnetic transmission to an RF compatible integrated circuit and is now being seen as a radical means of enhancing data handling process. RFID includes RFID readers, RFID tags and communication between the readers and the tags. RFID tags are categorized as either active or passive. Passive RFID readers interrogate small, battery-less transponders for their identity. The typical reading range is 1-2m. Active RFID tags are small transceivers that actively transmit their ID in reply to an interrogation. The range is larger usually up to tens of meters.

WLAN based systems operate in the $2.4 \mathrm{GHz}$ ISM band and provide bitrates of $11 \mathrm{Mbps}, 54 \mathrm{Mbps}$ and a range of $50-100 \mathrm{~m}$. WLAN has become very popular in public hotspots and enterprise locations during the last few years. Since WLAN infrastructure already exists, it is therefore appealing to use it for indoor location. Such systems provide an accuracy of approximately $2 \mathrm{~m}$. Bluetooth based systems are also operated in the $2.4 \mathrm{GHz}$ range. Compared to WLAN based sys- 


\begin{tabular}{|c|c|c|}
\hline Technology & Merits & Remarks \\
\hline Infrared & $\begin{array}{l}\text { Inexpensive (due to ubiquitous deployment) } \\
\text { Compact } \\
\text { Low power }\end{array}$ & $\begin{array}{l}\text { Typical range is upto } 5 \mathrm{~m} \\
\text { Restriction to line of sight conditions } \\
\text { Restriction to direct sunlight }\end{array}$ \\
\hline Ultrasound & $\begin{array}{l}\text { Relatively slow propagation } \\
\text { of speed of sound } \\
\text { allows for precise measurement } \\
\text { at low clock rates, making the system } \\
\text { simpler and inexpensive }\end{array}$ & $\begin{array}{l}\text { Typical range is } 3-10 \mathrm{~m} \text { have been reported } \\
\text { Environmental factors have substantial effects }\end{array}$ \\
\hline Radio Frequency & $\begin{array}{l}\text { Better than IR } \\
\text { interms of bandwidth, cost and speed }\end{array}$ & $\begin{array}{l}\text { No proper propagation model exists } \\
\text { Affected by multipath } \\
\text { Typical range of bluetooth is } 10-15 \mathrm{~m} \\
\text { Typical range of WLAN is } 50-100 \mathrm{~m} \\
\text { Typical range of RFID is } 1-10 \mathrm{~m} \\
\text { Typical range of cellular systems is } 100-150 \mathrm{~m}\end{array}$ \\
\hline DC Electromagnetic & $\begin{array}{l}\text { High precision } \\
\text { High signal propagation speed }\end{array}$ & $\begin{array}{l}\text { Typical range is } 1-3 \mathrm{~m} \\
\text { Signals are sensitive to environments } \\
\text { Precision calibration required, hence expensive } \\
\text { Difficult to install }\end{array}$ \\
\hline Optical & $\begin{array}{l}\text { High precision } \\
\text { Compact } \\
\text { Low power }\end{array}$ & $\begin{array}{l}\text { Typical range is upto } 5 \mathrm{~m} \\
\text { Restriction to line of sight conditions } \\
\text { Restriction to direct sunlight }\end{array}$ \\
\hline Inertial & & $\begin{array}{l}\text { Errors accumulate over time } \\
\text { Calibration }\end{array}$ \\
\hline UWB radio & $\begin{array}{l}\text { High precision and accuracy } \\
\text { Less affected by multipath } \\
\text { than the traditional RF systems }\end{array}$ & $\begin{array}{l}\text { Expensive } \\
\text { Higher receiver density than the } \\
\text { conventional RF systems } \\
\text { nevertheless its easier to install }\end{array}$ \\
\hline
\end{tabular}

Table 1. Enabling signal technologies

tems, they provide lower bitrates (up to $1 \mathrm{Mbps}$ ) and the range is shorter $(10-15 \mathrm{~m})$. Though Bluetooth was originally developed as a cable replacement technology, because of its ubiquitous connectivity it can also be used for location identification.

Wide area cellular based systems, locate the mobile users within the coverage area of the cell. A typical range covered by a cell is $100-150 \mathrm{~m}$.

UWB is based on sending ultra short pulses (typically $<1 n s)$. For location identification, UWB uses Time of Arrival measurement. The very short pulses lead to high accuracy and low power consumption.

\subsection{Distance Measuring Techniques}

The ranging technology forms the heart of any range based localization system. There are several rangebased techniques such as Time-of-arrival (TOA), Time difference of Arrival (TDOA), Angle-of-arrival (AOA), and Received Signal Strength Indication (RSSI). The TOA and TDOA make use of signal propagation time for finding the range of distance. To augment and complement TOA and TDOA, AOA was proposed. AOA allows nodes to estimate and map relative angles between the neighbors. However this approach requires costly antenna arrays on each node [33]. RSSI makes either theoretical or empirical calculations to convert the signal strength measurements to distance estimates.

On the other hand range free schemes make use of some algorithm that calculates the distance in terms of hop count to anchor nodes [12]. Few algorithms to mention using range free schemes are Centroid algorithm, DV-Hop, Amorphous, Point-In-Test (PIT) and Approximate Point-In-Test (APIT) [12]. In DV hop method, the anchor nodes are placed at known position and they transmit broadcast messages that are flooded throughout the network containing the location of the anchor node, and the distance between the anchors is obtained by using hop count as a metric. Later, any of the below discussed position estimation method is used to estimate the node location. The other range free schemes are explained in Section 5.2.

\subsection{Location Estimation Techniques}

The next step after the determination of the distance is the location estimation. There are different methods for estimating the location. Triangulation uses the geometric properties of triangles to compute object locations. It is a positioning procedure that relies on angle measurements with respect to the known landmarks. Triangulation is subcategorized into lateration - using distance measurements - and angula- 
tion - using angle or bearing measurements [13]. Trilateration uses ranges to at least three known node position to find the coordinates of unknown nodes. The trilateration [26] procedure starts with an a priori estimated position that is later corrected towards the true position. There are many other types of lateration such as Atomic multilateration and Collaborative multilateration, which are addressed in detail in [34]. Triangulation has been extensively used for both navigation and geodetic purposes, because measuring angles is easier and more accurate than measuring distances. Also transformation from triangulation to trilateration is possible with simple transformation methods [38]. Proximity measures the nearness to a known set of points. The objects presence is sensed using the physical phenomenon with limited range. Scene analysis examines a view from a particular vantage point to draw conclusions about the observer's location [13][17]. The scene itself can contain visual images, such as frames captured by a wearable camera or any other measurable physical phenomena, such as electromagnetic characteristics that occur when an object is at a particular position and orientation.

When localization problem is addressed in wireless sensor networks, there is yet another type of location estimation method called min-max algorithm [19] The main idea is to construct a bounding box for each anchor using its position and distance estimate, and then to determine the intersection of these boxes. The intersection of the bounding boxes is computed by taking the maximum of all coordinate minimums and the minimum of all maximums. Hence it is names as minmax. The estimated position by min-max is found to match closely with the true position computed through lateration.

Least square algorithm [33] [9] is also used to derive position estimation from collection of reference points and their associated ranges. The existing location systems use techniques and technologies described above.Figure 1 shows that there are 3 steps involved in any localization system namely, distance measurement to anchors or beacons, position estimation and position refinement, which is an optional step [19]. However, the choice of the techniques and technology affects the granularity and accuracy of the location information.

\section{State of the Art}

\subsection{Implemented Systems}

Loran was the first navigation system, launched before the World War II to employ time difference of

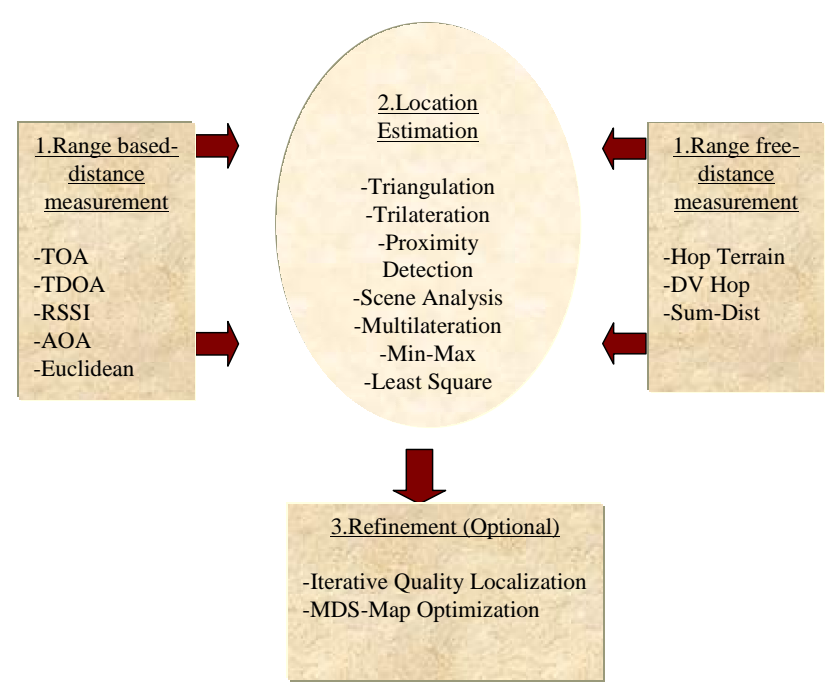

Figure 1. Steps in localization

arrival of radio signals, developed by MIT radiation lab [28]. It was also the first true all weather position finding system, but it is only 2 dimensional. Transit was the first operational satellite based navigational system launched in the year 1959. Transit users determine their position on earth by measuring the Doppler shift of signals transmitted by the satellites. Global Positioning Systems [28] is one of the oldest location technologies that provided the location of the users in 3D. It works well in urban outdoor environments, but they are quirky, unreliable and their accuracy degrades when the device is indoors and has limited line-of-sights to the satellites. It is not ubiquitously available and hence not suitable for under water and cluttered urban environments [5]. Additionally, this cannot be used for the future calm technology, as it is not practically implementable on low power devices and also size and the cost poses harsh limitations. Apart from GPS, there are also other systems, which are used, in the outdoor environment mainly for navigation applications. Table 2 below shows the comparison of various outdoor geo-location systems.

Bulusu has designed a GPS-less system for outdoor locations suited for very small, low cost devices [21]. The system uses a RF-based signal technology and proximity based position estimation and got satisfying results. However Bulusu suggests many areas of improvements like adding robustness to the system, also adapting to noisy environments. In principle the system can be used indoors, but in this case the accuracy is bad.

The active badge [37] is the one of the early centralized indoor personal location system making use 


\begin{tabular}{|c|c|c|c||c|}
\hline System & Method & Coverage & Dimensions & Accuracy \\
\hline \hline Loran & Hyperbolic & Cont & $2 \mathrm{D}$ & $250 \mathrm{~m}$ \\
\hline Transit & Doppler shift & Global & $2 \mathrm{D}$ & $25 \mathrm{~m}$ \\
\hline Omega & Hyperbolic & Global/cont & $2 \mathrm{D}$ & $2-4 \mathrm{~km}$ \\
\hline GPS & Spherical & GLobal/Cont & $3 \mathrm{D}$ & $5-10 \mathrm{~m}$ \\
\hline
\end{tabular}

\section{Table 2. A comparison of several radio navigation methods}
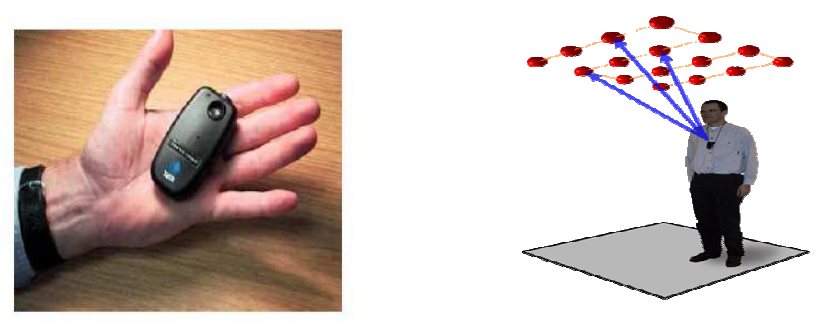

Figure 2. Active Bat System,developed by AT\&T and Cambridge University

of IR technology. Each person in the office wears a badge, which emits a unique IR signal that is then gathered by the network of sensors and collected by a master server. The information is then relayed, to the visual display location manager. Interestingly, its development was not driven by the long-term vision of ubiquitous computing, but by a very practical concern, of how to track people in office setting and to route telephone calls. This was mainly used as an aid for the telephone receptionists to direct the phone calls to appropriate persons during working time. Though it was providing significant number of advantages, making use of IR has its own drawbacks. The range was limited and obstacles such as walls and windows made it difficult for the IR signal to propagate. Nevertheless it works well for the intended application of routing telephone calls.

However, the Active Badge is not able to provide fine-grained 3D location information, which is needed by many applications. So the Active Bat [10] system was developed, with a primary focus on low power, low cost and accuracy. The Bat is attached to the objects or persons whose location has to be determined. These bat transmitters emit ultrasound pulse, which are received by the receiver that is mounted on the ceiling. A central controller coordinates the transmitters and receivers. To locate a particular bat, the controller sends the unique ID over the radio channel. When a bat detects its ID, it sends an ultrasound pulse, which is picked by receivers in the ceiling. From the time-of- flight measurements, the system can calculate the 3D position of a bat to an accuracy of $3 \mathrm{~cm}$.

Cricket location [31] support systems make use of proximity based lateration techniques for providing location information. User privacy, decentralized administration, maintaining network heterogeneity, cost and granularity were its primary design goals. Many beacons were installed at known locations, which advertise the identity of that space with the use of some character string, every device in the network has a listener attached to it. Listener uses some inference algorithm to determine the space in which they are currently located by listening to the beacon announcements. Each beacon sends two signals, an RF signal carrying the location data and an ultrasound carrying a narrow pulse. Based on the difference of arrival times, the device finds the absolute distance between the beacon and the listener.

$R A D A R$ makes use of $\mathrm{RF}$ signal for finding the user location [29]. The design goal of RADAR is to complement the data networking capabilities of RF WLANs with accurate user location capabilities, thereby boosting the value of such networks. RADAR was intended for indoor applications. A centralized system gathers signal strength information from multiple receivers and performs triangulation to compute the location of the user.

Pinpoint $3 D-i D$ [2] is similar to RADAR, but expensive. Proprietary base stations and tag hardware are used to measure radio time of flight. It uses an installed array of antennas at known positions to perform multilateration. Pinpoint's accuracy is roughly 1 to 3 meters.

In the SpotOn system [14], special tags use radio signal attenuation to estimate the distance between tags. The aim in SpotOn is to localize a wireless device relative to one another, rather than to fixed base stations, allowing for ad-hoc localization.

HP Labs SmartLOCUS [16] uses synchronized $\mathrm{RF}$ and ultrasound differential time-of-flight measurements to determine the inter-nodal range between any two nodes. With a multitude of nodes in the systems, a distributed localization algorithm operates on the obtained ranges to create a self-organizing coordinate system. It yields an accuracy of $2-15 \mathrm{~cm}$. 


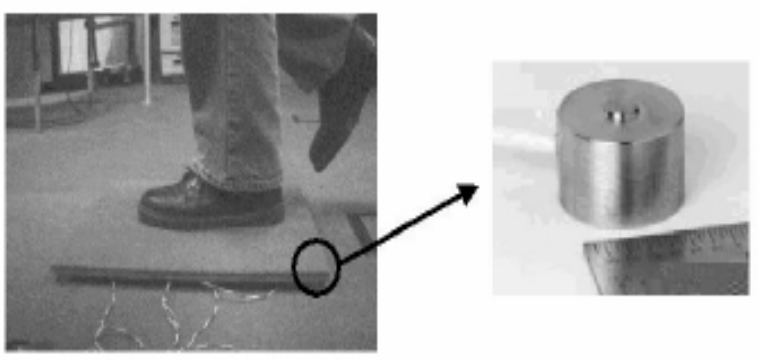

Figure 3. Smart Floor Plate (left) and load cell (right)

Georgia Institute of Technology's Smart floor [27] identifies people based on their footsteps. The goal of the system is to create an accurate system for recognizing a user's identity. Figure 3 shows the smart floor plate. However this technologies negative side is the huge installation cost and infrastructure cost.

UBISENSE [30], uses UWB technology to locate people and objects to an accuracy of $15 \mathrm{~cm}$. Sensors are mounted in the area to be monitored. Ubitags attached to objects or carried by people are then automatically tracked. Indoor tracking is a difficult problem and UBISENSE combines UWB technology and a unique algorithm to combat these harsh indoor effects and provide accuracy and reliability.

There are also systems that make use of Radio Frequency IDentification (RFID) technology, for locating objects inside buildings. RFID is a means of storing and retrieving data through electromagnetic transmission to an RF compatible integrated circuit and is now being seen as a radical means of enhancing the supply chain, especially for Returnable Transport Items [4]. RFID includes RFID readers, RFID tags and communication between them. RFID tags are categorized as either active or passive. A passive tag, with no power source can be activated by a reader device that transmits an energy signal to the tag. The tags themselves consist of antennae connected to a silicon chip. Active RFID tags are powered by an internal battery and the tag data can be rewritten and/or modified. Passive tags are consequently much lighter than active tags, less expensive and may offer a virtually unlimited operational lifetime. The trade off is that they have shorter read ranges than active tags and require a higher-powered reader. The no contact and non lineof-sight nature of this technology offers promises. One example is the $L A N D M A R C$ systems [23].
Computer vision has also been used in localization. Microsoft's research on Easy Living [17] uses Digiclops real-time 3D cameras to provide stereovisionpositioning capability in a home environment. However the dependence on infrastructural processing power, can limit the scalability of such systems for many applications. Ekahau Positioning system is software based positioning solution that can continuously pinpoint and track the location of mobile computing devices with an accuracy of 1-2 meters in indoor and campus environments. Unlike the competing solutions, Ekahau technology does not require any additional wireless infrastructure on top of the standard Wi-Fi network [1].

All the systems described above are already implemented. However, there are many other theoretical methods available to solve the location identification problem. We describe these methods in the next section.

\subsection{Theoretical Methods}

An often ignored issue in ongoing research is the impact of beacon density and the placement of the beacons. Self-configuring localization systems consider beacon density as an important parameter in characterizing the localization quality. Two algorithms were developed HEAP and STROBE [5] depending on the density of beacons in the network. The goal of HEAP is incremental beacon placement, meaning that new beacons can be added to the network without the need of re-deployment, by constantly checking the localization error. In STROBE, the functionality among the beacons is rotated among themselves by turning them on and off selectively. This is particularly useful for large networks whose primary goal is to have unattended operation.

In the convex optimization [20] approach, the positional information is inferred from connectivity imposed proximity constraints. Few nodes have known locations, called the anchor nodes, and the remaining nodes infer their position from the knowledge about communication links. This method requires a centralized computation. For the technique to work well the anchor nodes must be placed in the outer boundary, preferably at the corners. When all anchors are located in the interior of the network, the position estimation of the outer node collapses towards the center, giving rise to large positional errors

$M D S-M A P[35]$ is a method that makes use of connectivity information to provide locations in a network with or without beacons (known co-ordinates). The advantage of MDS-MAP is that it has a wide range of 
applicability, having the ability to work with both simple connectivity and range measurements to provide both absolute and relative positioning [35] [25]. Both convex optimization and MDS-MAP requires centralized computation.

Whilst lot of research was initially based on rangebased schemes, many developments in range free schemes also came in. Range free schemes make no assumption about validity of distance or angle information like the range based schemes. Some examples to quote are Centroid algorithm, APIT, amorphous localization and DV-Hop algorithm. In the centroid method [21], each node estimates its location by calculating the center of the locations of all seeds (or anchors) it hears. If seeds are well positioned, the location error can be reduced [6], but this is not possible in ad hoc deployments. The APIT method [12] isolates the environment into triangular regions between beaconing nodes, and uses a grid algorithm to calculate the maximum area in which a node will likely reside. DV based positioning algorithms are localized, distributed, hop by hop positioning algorithms [25] [26]. They work as an extension of both distance vector routing and GPS positioning in order to provide approximate positions for all nodes in a network where only a limited fraction of nodes have self positioning capabilities. They use the same principle of GPS, with the difference that the landmarks are contacted by hop-by-hop fashion rather than a direct connection and similar to distance vector each node at any time can communicate only with its neighbors. The amorphous method [22] is similar to DV-hop as the coordinates of the seeds are flooded throughout the network so each node can maintain a hop count to that seed. Nodes calculate their position based on the received seed locations and corresponding hop count. From an extensive study on range free schemes and on benchmarking various range free schemes, TianHe etal. [12] concluded that range free schemes offers cost effective solutions. Table 3 lists the comparison between various range free schemes. In TianHe etal. experiments several parameters such as node density (ND), anchors heard ( $\mathrm{AH})$, anchor to node range ratio (ANR), anchor percentage (AP), degree of irregularity (DOI), GPS error and placement of node and anchors were investigated.

Recently research on localization is focused on incorporating the mobility model. Although mobility would make the analysis more difficult, more accuracy is obtained. In [15], Lingxuan $\mathrm{Hu}$ etal. use a sequential Monte Carlo Localization method and argues that they exploited mobility to improve accuracy and precision of localization. Probabilistic techniques, such as Markov modeling, Kalman filtering and Bayesian analysis can also be used to determine the absolute location of a mobile node [18].

Table 4 gives a global view of localization techniques classified by achievable accuracy and the type of location estimation used for various technologies [11].

\section{Conclusions and Future work}

Designing a location system for a particular environment presents difficulties when the system is applied to other environments. Despite the plethora of established location technology, there is no single location technology that may be relied upon in all environments to provide accurate location information. Clearly "No one size fits all", there may not be a single best technology. However each of the techniques has its own pros and cons.

Ubiquitous computing is the wave of the future. Recent advancement in the various related technologies are paving way for the design and implementation of the future ubiquitous computing. Location identification is an important research area that had gained additional credit since the epoch of pervasive computing. This paper provided an overview of the existing location systems/algorithms and also highlighted the limitations of the existing technologies. In order to improve the existing techniques in future, we would focus on developing new distributed localization algorithms for resource poor ambient systems which addresses specific issues like the ease of deployment, scalability, automatic configuration providing easy adaptability to different types of environment, self-calibration, responsiveness, accuracy etc keeping an eye on the cost factor (computational power, resource needed and money) and should be tolerant to node failures and range errors. Also of great importance is the selforganization as it is infeasible to manually configure the location determination processes for a large number of mobile devices in random configurations with random environmental characteristics.

While there is plenty of research going on in developing new systems or algorithms, yet another available solution on hand is to make use of the existing systems/algorithms and choose the best in each case and fuse the location information reported by several technologies to get more meaningful results. It is not just sufficient to have technology development, also of foremost importance is to use the existing systems and have a means to have them integrated so that seamless transition between the available systems is achieved. Ideally the localization should provide a framework to integrate location reading from all these sensor types 


\begin{tabular}{|c|c|c|c||c|}
\hline & Centroid & DV Hop & Amorphous & APIT \\
\hline Accuracy & Fair & Good & Good & Good \\
\hline Node Density & $>10$ & $>8$ & $>8$ & $>6$ \\
\hline Anchors Heard & $>10$ & $>8$ & $>8$ & $>10$ \\
\hline ANR & $>0$ & $>0$ & $>0$ & $>3$ \\
\hline DOI & Good & Good & Fair & Good \\
\hline GPSError & Good & Good & Fair & Good \\
\hline Overhead & Smallest & Largest & Large & Small \\
\hline
\end{tabular}

Table 3. Comparison of range free schemes

\begin{tabular}{|c|c|c|c|}
\hline Technology & Accuracy & Location Estimation & Example \\
\hline Infrared based & $5-10 \mathrm{~m}$ & Proximity & Active Badge \\
\hline Ultrasound based & $1-10 \mathrm{~cm}$ & TOF-lateration & Active Bat \\
\hline Vision based & $1 \mathrm{~cm}-1 \mathrm{~m}$ & Scene Analysis & Easy Living \\
\hline RF-UWB based & $6-10 \mathrm{~cm}$ & TOF-triangulation & \\
\hline RF-Bluetooth based & $2-10 \mathrm{~m}$ & Proximity, Triangulation & Radar \\
\hline RF-WLAN based & $2-100 \mathrm{~m}$ & Triangulation,Proximity and Scene Analysis & GPS \\
\hline Satellite based & $5-10 \mathrm{~m}$ & Triangulation & GSM localization \\
\hline RF wide area cellular based & $50-10 \mathrm{~m}$ & Triangulation and Proximity & Landmarc \\
\hline RFID based & $5 \mathrm{~cm}-5 \mathrm{~m}$ & Proximity & \\
\hline
\end{tabular}

Table 4. Summary of existing localization systems

into one seamless environment. The future research in the project Smart Surroundings [3] addresses all these issues and will provide an open platform for supporting new architectures and frameworks for the future ambient systems.

\section{References}

[1] Ekahau positioning system.

[2] Pinpoint 3D positioning system.

[3] The smart surroundings project.

[4] Making waves: Rfid adoption in returnable packaging, 2004.

[5] N. Bulusu. Self-Configuring Location Systems. $\mathrm{PhD}$ thesis, University of California, Los Angeles, 2002.

[6] N. Bulusu, D. Estrin, L. Girod, and J. Heidemann. Scalable coordination for wireless sensor networks: Self-configuring localization systems. In Proceedings of the Sixth International Symposium on Communication Theory and Applications (ISCTA '01), page xxx. University of California, Los Angeles, July 15-20th 2001.

[7] A. K. Dey, D. Salber, and G. D. Abowd. A conceptual framework and a toolkit for supporting the rapid prototyping of context-aware appli- cations. Human-Computer Interaction Journal, 16(2-4):97-166, 2001.

[8] D. Estrin, D. Culler, K. Pister, and G. Sukhatme. Connecting the physical world with pervasive networks. Pervasive Computing, 1(1):59-69, 2002.

[9] L. Evers, S. Dulman, and P. Havinga. A distributed precision based localization algorithm for ad-hoc networks. In Proceedings of the Second International Conference on Pervasive Computing (PERVASIVE 2004), volume 3001 of Lecture Notes in Computer Science, pages 269-286. Springer Verlag, Berlin, Apr. 2004.

[10] A. Harter and A. Hopper. A distributed location system for the active office. IEEE Network, 8(1):62-70, Feb. 1994

[11] M. Hazas, J. Scott, and J. Krumm. Locationaware computing comes of age. IEEE Computer Magazine, 37(2):95-97, Feb. 2004.

[12] T. He, C. Huang, B. M. Blum, J. A. Stankovic, and T. Abdelzaher. Range-free localization schemes for large scale sensor networks. In Proceedings of the 9th annual international conference on Mobile computing and networking (MobiCom'03), pages 81-95. ACM Press, 2003.

[13] J. Hightower and G. Borriello. Location systems for ubiquitous computing. Computer, 34(8):57$66,2001$. 
[14] J. Hightower, R. Want, and G.Borriello. SpotON: An indoor 3D location sensing technology based on RF signal strength. Technical Report UWCSE 00-02-02, University of Washington,Seattle, February 2000.

[15] L. Hu and D. Evans. Localization for mobile sensor networks. In Proceedings of the Tenth Annual International Conference on Mobile Computing and Networking (MobiCom 2004), Sept. 2004.

[16] A. Jiminez, F. Seco, R. Cere, and L. Calderon. Absolute locatilization using active beacons :a survey and iai-csic contributions.

[17] J. Krumm, S. Harris, B. Meyers, B. Brumitt, M. Hale, and S. Shafer. Multi-camera multiperson tracking for easyliving. In VS '00: Proceedings of the Third IEEE International Workshop on Visual Surveillance (VS'2000), page 3. IEEE Computer Society, 2000.

[18] A. M. Ladd, K. E. Bekris, A. Rudys, L. E. Kavraki, D. S. Wallach, and G. Marceau. Robotics-based location sensing using wireless ethernet. In MobiCom '02: Proceedings of the 8th annual international conference on Mobile computing and networking, pages 227-238. ACM Press, 2002.

[19] K. Langendoen and N. Reijers. Distributed localization in wireless sensor networks: a quantitative comparison. Comput. Networks, 43(4):499-518, 2003.

[20] L.Doherty, K.Pister, and L. Ghaoui. Convex position estimation in wireless sensor networks. In IEEE INFOCOM, Apr. 2001.

[21] D. E. N. Bulusu, J. Heidenmann. GPS-less low cost outdoor localization for very small devices. IEEE Personal Communications Magazine, 7(5):28-34, October 2000.

[22] R. Nagpal, H. Shrobe, and J. Bachrach. Organizing a global coordinate system from local information on an ad hoc sensor network. In Proceedings of the 2nd International Workshop on Information Processing in Sensor Networks (IPSN '03), number 2634 in LNCS. Springer Verlag, Berlin, Apr. 2003.

[23] L. M. Ni, Y. Liu, Y. C. Lau, and A. P. Patil. LANDMARC: Indoor location sensing using active rfid. Wireless Networks. Special Issue on Pervasive Computing and Communications, 10(6):701-710, 2004.
[24] D. Niculescu. Positioning in ad hoc sensor networks. IEEE Network, 18(4), July 2004.

[25] D. Niculescu and B. Nath. Dv based positioning in ad hoc networks. Telecommunication Systems, 22(1-4):267-280, 2003.

[26] D. Niculescu and B. Nath. Position and orientation in ad hoc networks. Elsevier journal of $\mathrm{Ad}$ Hoc Networks, 2(2):133-151, Apr. 2004.

[27] R. J. Orr and G. D. Abowd. The smart floor: a mechanism for natural user identification and tracking. In CHI '00: $C H I$ 'OO extended abstracts on Human factors in computing systems, pages 275-276. ACM Press, 2000.

[28] S. Pace, G. Frost, I. Lachow, D. Frelinger, D. Fossum, D. K. Wassem, and M. Pinto. The Global Positioning System, chapter GPS history, chronology and budgets, pages 237-270. RAND Coorporation, 1995.

[29] P.Bahl and V.Padmanabhan. RADAR: An inbuilding RF based user location and tracking system. In IEEE Infocom, volume 2, pages 775-784, March 2000.

[30] PeteSteggles and JayCadman. A comparison of rf tag location products for real world applications. Technical report, Ubisense limited, Mar. 2004.

[31] N. B. Priyantha, A. Chakraborty, and H. Balakrishnan. The cricket location-support system. In Proceedings of the 6th annual international conference on Mobile computing and networking (MobiCom'00), pages 32-43. ACM Press, 2000.

[32] C. Savarese, J. Rabaey, and J. Beutel. Locationing in distributed ad-hoc wireless sensor networks. In $I C A S S P$ 2001, 2001.

[33] C. Savarese, J. M. Rabaey, and K. Langendoen. Robust positioning algorithms for distributed adhoc wireless sensor networks. In Proceedings of the General Track: 2002 USENIX Annual Technical Conference, pages 317-327. USENIX Association, 2002 .

[34] A. Savvides, C.-C. Han, and M. B. Strivastava. Dynamic fine-grained localization in ad-hoc networks of sensors. In Proceedings of the 7th annual international conference on Mobile computing and networking (MobiCom'01), pages 166-179. ACM Press, 2001. 
[35] Y. Shang and W. Ruml. Improved mds-based localization. In Proceedings of the 23rd Conference of the IEEE Communicatons Society (Infocom 2004), Hong Kong, 2004.

[36] Y. Shang, W. Ruml, Y. Zhang, and M. P. J. Fromherz. Localization from mere connectivity. In Proceedings of the 4 th ACM international symposium on Mobile ad hoc networking \& computing (MobiHoc'03), pages 201-212. ACM Press, 2003.

[37] R. Want, A. Hopper, V. Falc\&\#227;o, and J. Gibbons. The active badge location system. ACM Trans. Inf. Syst., 10(1):91-102, 1992.

[38] M. Weiser. The computer for the twenty-first century. Scientific American, pages 94-104, Sept. 1991.

[39] K. Whitehouse. The Design of Calamari: an Ad-hoc Localization System for Sensor Networks. Master's thesis, University of California at Berkeley, 2002. 